\title{
EL PRINCIPIO DE CONFIANZA COMO CRITERIO DELIMITADOR DE LA RESPONSABILIDAD PENAL DE LOS MÉDICOS
}

\section{Lautaro Camilo Contreras Chaimovich ${ }^{1}$}

Resumen: En el presente artículo el autor analiza los presupuestos y límites del principio de confianza como criterio de concreción del cuidado debido exigible a los médicos. Para ello, se distingue entre división horizontal y vertical del trabajo. En el ámbito de la división horizontal, se destaca la relevancia que tiene una clara delimitación de ámbitos de responsabilidad para la vigencia del principio de confianza. En el plano de la división vertical, se explica la delegación de actividades médicas en enfermeras y auxiliares paramédicos (contemplada en el inc. $2^{\circ}$ del art. 113 del Código Sanitario), así como los deberes de elección, instrucción y supervigilancia que han de cumplirse para que los facultativos puedan confiar en la correcta ejecución de las tareas delegadas.

Palabras claves: responsabilidad penal médica, principio de confianza, delegación de actividades médicas.

\section{The principle of reliance as a criterion for delimiting the criminal responsibility of physicians}

\begin{abstract}
In the present article, the author analyzes the pre-requisites and boundaries of the principle of reliance as a criterion for determining the due care that can be demanded from physicians. For that purpose, a distinction is made between horizontal and vertical division of labour. Regarding horizontal division, the relevance of a clear delimitation of scopes of responsibility for the principle of reliance to apply is highlighted. Regarding vertical division, an explanation about delegation of medical activities to nurses and paramedic auxiliaries (contemplated in indent 2 of article 113 of the Sanitary Code) is offered, as well as one about the duties of selection, instruction and supervision that must be fulfilled for the physicians to be allowed to trust in the correct execution of the delegated tasks.
\end{abstract}

Keywords: criminal responsability of physicians, principle of reliance, delegation of medical activities

\section{O princípio da confiança como critério delimitador da responsabilidade penal dos médicos}

Resumo: no presente artigo o autor analisa os pressupostos e limites do princípio da confiança como critério de materializaçáo do cuidado devido exigido dos médicos. Para isso se distingue entre divisão horizontal e vertical do trabalho. No âmbito da divisão horizontal se destaca a relevância que tem uma clara delimitação de âmbitos de responsabilidade para a vigência do princípio da confiança. No plano vertical se explica a delegaçáo de atividades médicas para enfermeiras e auxiliares paramédicos (contemplada no parágrafo $2^{\circ}$ do artigo 113 do Código Sanitário), assim como os deveres de eleição, instrução e supervigilância que se tem de cumprir para que os médicos possam confiar na correta execução das tarefas delegadas.

Palavras-chave: responsabilidade penal médica, princípio da confiança, delegação de atividades médicas

\footnotetext{
${ }^{1}$ Facultad de Derecho, Universidad de Chile, Chile

Correspondencia: lcontreras@derecho.uchile.cl.
} 


\section{Introducción}

El impresionante desarrollo que ha experimentado la medicina en las últimas décadas, así como la cada vez mayor especialización y subespecialización de los profesionales que se desempeñan en el área de la salud, hacen indispensable la actuación en división del trabajo(1:34). Incluso en un procedimiento médico relativamente sencillo, como una apendicectomía, interviene un equipo conformado por varias personas, que cumplen distintas funciones.

Las ventajas de la división del trabajo para un adecuado diagnóstico y tratamiento son indiscutibles, al permitir aprovechar los conocimientos y experiencias de sujetos altamente especializados en distintas áreas. Sin embargo, la división de labores también puede generar importantes riesgos para el paciente, derivados de una falta de coordinación al interior del equipo médico, de errores de comunicación entre sus integrantes, de una delegación de tareas a personas no suficientemente calificadas, etcétera. Tales peligros pueden realizarse en un resultado lesivo, lo que permitirá, dado el caso, fundar una responsabilidad penal a título de homicidio o lesiones imprudentes.

Ahora bien, sancionar a cada uno de los miembros de un equipo médico, por la lesión al cuidado debido en que hayan incurrido los otros, no sería conciliable con la necesaria diferenciación y especificación de los deberes de conducta exigibles en el ámbito de la salud (esa diferenciación y especificación es indispensable, por lo demás, para una adecuada conservación de los bienes jurídicos del paciente). Por eso, la responsabilidad penal de cada miembro del equipo debe limitarse a su propio ámbito de competencia $(2)^{2}$. Tal limitación surge de la observancia de los principios de estricta división del trabajo y de confianza(3:368). Según el principio de confianza, cuyo origen y desarrollo se encuentra en el Derecho penal alemán,

\footnotetext{
${ }^{2}$ Algo distinto puede inferirse de la conocida sentencia de la Corte Suprema de 20 de junio de 1996, Rol n. 33.393-1995(2), en la cual se condenó por lesiones imprudentes a todos los miembros de un equipo médico (cirujanos, anestesista, pabellonera y arsenalera), por haber realizado una ortoplastía total de la cadera izquierda de un paciente, en circunstancias que la cadera que debía ser operada era la derecha. Esta sentencia desconoce que los deberes de cuidado que pesan sobre las distintas personas que intervienen en una cirugía no son idénticos para todas ellas, sino que tienen un contenido diverso según la función que cada una cumpla en el procedimiento médico.
}

cada integrante de un equipo médico puede confiar en que los restantes miembros realizarán sus tareas con el cuidado exigido, a menos que existan indicios en un sentido contrario.

La finalidad del presente artículo consiste en analizar los presupuestos y límites del principio de confianza como criterio de concreción del cuidado debido que los médicos tienen que cumplir. Si bien se han publicado en nuestro país algunos trabajos que abordan estas cuestiones(4), aún existen aspectos no explorados, tales como el presupuesto de la vigencia de la confianza entre médicos de distintas especialidades, los requisitos que deben reunirse para que un facultativo confíe en que el personal de colaboración ejecutará correctamente una actividad médica delegada, o los problemas de imputación objetiva que se pueden presentar cuando se infringe el denominado "deber de supervigilancia" (a que alude el inc. $2^{\circ}$ del art. 113 del Código Sanitario). El presente artículo se abocará, precisamente, al estudio de tales aspectos.

\section{La distinción entre división horizontal y ver- tical del trabajo}

Una correcta comprensión de los presupuestos y límites del principio de confianza exige distinguir necesariamente entre división horizontal y vertical del trabajo. Por división horizontal del trabajo debemos entender la actuación conjunta de personas jerárquicamente independientes, que no poseen la facultad de darse instrucciones u órdenes unas a otras. Esta clase de división de labores existe frecuentemente entre médicos de distintas especialidades, entre médicos y farmacólogos clínicos, entre cirujanos y jefes de bancos de sangre, etcétera. En cambio, por división vertical del trabajo debemos entender la intervención conjunta de personas que tienen vínculos de subordinación entre sí, estando dotada alguna o algunas de ellas del poder de dar instrucciones a la o las demás. Ejemplo paradigmático de división vertical del trabajo es la existente entre médico y enfermera, o entre enfermera y auxiliar paramédico ${ }^{3}$.

\footnotetext{
${ }^{3}$ Según el art. $2^{\circ}$ del Reglamento para el ejercicio de las profesiones auxiliares de la medicina, odontología, química y farmacia y otras, "auxiliar paramédico" es la persona habilitada para ejecutar técnicas y procedimientos, labores de apoyo diagnóstico y terapéutico, y otras actividades que se les asigne en el ámbito de su competencia, bajo la supervisión, control y dependencia del profesional de la salud correspondiente.
} 
A continuación, expondremos los presupuestos y límites del principio de confianza en la división horizontal del trabajo, y posteriormente lo haremos respecto de la división vertical.

\section{División horizontal del trabajo}

\subsection{Vigencia del principio de confianza en la división horizontal del trabajo}

Respecto de la actuación conjunta de médicos que no tienen un vínculo de subordinación entre sí y que pertenecen a distintas especialidades, la jurisprudencia comparada ha planteado que aquellos pueden confiar en que sus colegas cumplirán sus tareas con el cuidado debido(5:650). Si existiera una obligación de supervigilancia respecto de la labor que desempeñan los otros, la intervención de distintos especialistas en división horizontal del trabajo perdería todo sentido y se crearían riesgos innecesarios para los bienes jurídicos del paciente, al no poder concentrarse cada facultativo en sus propias tareas (5:650). Por eso, y a modo de ejemplo, el Derecho no puede exigir a un cirujano que, en su calidad de no especialista, vigile y controle el tratamiento anestésico que un especialista (anestesiólogo) realiza. En consecuencia, si el paciente muere a causa de la infracción de un deber jurídico cuyo cumplimiento se situaba en el ámbito de competencia exclusivo del anestesista, solo este podría ser castigado por homicidio culposo, aunque la conducta del cirujano haya sido causal (en el sentido de la teoría de la equivalencia de las condiciones) para el resultado.

En relación con la intervención conjunta de cirujanos y anestesistas en un procedimiento quirúrgico, que representa el caso prototípico de la división horizontal del trabajo en el ámbito médico, es necesario establecer cuál es la esfera de competencia de cada uno. En la etapa de cuidados preoperatorios, el anestesiólogo es el responsable de constatar la capacidad del paciente para resistir el tipo de anestesia que le pretende suministrar; además, debe comprobar que el enfermo está en ayunas, para así evitar una neumonía por aspiración(6:136). Por su parte, al cirujano le corresponde decidir si es procedente realizar la intervención y cuándo esta debe tener lugar(3:372). En la fase de cuidados intraoperatorios el cirujano es responsable por la intervención quirúrgica misma y los riesgos derivados de ella, mientras que el anestesiólogo debe vigilar y mantener las funciones vitales del paciente durante la operación(7:113). Por último, en la etapa de cuidados postoperatorios, el anestesiólogo tiene el deber de seguir vigilando al paciente hasta que sus funciones vitales se desarrollen con normalidad y se recupere por completo de los efectos de la anestesia; en cambio, el control de los riesgos derivados de la cirugía misma, como las hemorragias postoperatorias, forma parte del ámbito de responsabilidad del cirujano. Los ámbitos de competencia de cirujanos y anestesistas, recién descritos, pueden sufrir modificaciones en caso de que estos profesionales deleguen determinadas actividades de las que son responsables en terceros. Así, por ejemplo, un anestesiólogo puede delegar los cuidados postoperatorios en el personal de enfermería que se desempeña en la unidad de recuperación anestésica ${ }^{4}$.

\subsection{Presupuesto de la vigencia del principio de confianza en la división horizontal del trabajo}

La vigencia del principio de confianza en la división horizontal del trabajo exige siempre una clara delimitación de ámbitos de responsabilidad(8:4). $\mathrm{Y}$ es que la confianza en que un colega, respecto del cual no hay una relación de subordinación, cumplirá su tarea con el cuidado debido solo puede existir cuando a aquel le compete precisamente realizar esa tarea(9:25). Por eso, si hay labores respecto de las cuales existen conflictos negativos de competencia, ya no habrá confianza que rija y todos los médicos tratantes serán responsables por su correcta realización.

La delimitación de ámbitos de responsabilidad en la división horizontal del trabajo puede tener su origen en la organización interna de la respectiva clínica u hospital, fijada, por ejemplo, a través de protocolos escritos(10:58ss.). Estos protocolos permiten conocer, justamente, quién es el competente para realizar una determinada tarea o labor, en cuya ejecución, entonces, se puede confiar. Otra fuente que sirve para delimitar los ámbitos de responsabilidad en la división horizontal de la labor médica son las reglas extrapenales. Así, por

\footnotetext{
${ }^{4}$ En este caso, estaremos frente a un supuesto de delegación de actividades médicas en personal de colaboración, cuestión tratada en la sección 3 del presente trabajo.
} 
ejemplo, la Norma General Técnica n.o 51 del Ministerio de Salud, sobre radioterapia oncológica, indica que corresponde al médico oncólogo radioterapeuta (y no a otro facultativo) aplicar la o las terapias para mitigar los síntomas secundarios que los pacientes sufran(11:18). La delimitación de esferas de competencia también puede surgir a partir de la asignación de roles que tiene lugar en el seno de las ciencias médicas, a través de la especialización y profesionalización, y que se manifiesta por medio de reglas extrajurídicas y planes de estudios diferenciados para distintos médicos especialistas(12:351). Así ocurre, por ejemplo, tratándose de cirujanos y anestesistas a quienes la propia medicina asigna funciones y ámbitos de responsabilidad diferenciados. Por último, la delimitación de esferas de responsabilidad también puede verificarse mediante acuerdos entre los profesionales que intervienen en el diagnóstico o tratamiento de un determinado paciente. La adopción de estos acuerdos es legítima, siendo una manifestación del ejercicio de la libertad de terapia médica(9:48ss.).

\subsection{Excepciones al principio de confianza en la división horizontal del trabajo}

El principio de confianza deja de regir en la división horizontal del trabajo cuando, en la situación concreta, es cognoscible que el colega no está en condiciones de cumplir correctamente con la labor que le compete (por ejemplo, a causa del agotamiento provocado por un turno extenuante), o que está infringiendo o infringirá la lex $\operatorname{artis}(7: 106 s s$.$) . La confianza tampoco ope-$ ra en favor de aquel médico que ha quebrantado sus propios deberes de cuidado; el facultativo que no se ha comportado correctamente ya no puede esperar que los demás actúen según el cuidado debido(13:321ss.). Sin embargo, esto no significa que todo resultado de muerte o lesión corporal, que haya tenido lugar después del quebrantamiento de un deber de cuidado, funde siempre un supuesto de responsabilidad penal por homicidio o lesiones imprudentes. Esto solo ocurrirá si se verifica la necesaria relación de contrariedad al deber, es decir, si un comportamiento del médico ajustado a la lex artis hubiera evitado con una probabilidad rayana en la certeza el resultado lesivo(14:166ss.).

\section{División vertical del trabajo}

\subsection{La delegación de actividades médicas en personal de colaboración}

La vigencia del principio de confianza en la división vertical del trabajo cobra especial relevancia en los supuestos de delegación de actividades médicas a enfermeras o a auxiliares paramédicos, esto es, en los casos en que un facultativo asigna, por medio de una instrucción, determinadas tareas propias de la profesión médica al personal de colaboración(15:54). La delegación de actividades médicas está reconocida en el inc. $2^{\circ}$ del art. 113 del Código Sanitario, según el cual quienes cumplen funciones de colaboración médica pueden realizar algunas actividades de diagnóstico, pronóstico o tratamiento de pacientes, siempre que medie la indicación y supervigilancia de un facultativo.

Las ventajas de la delegación son evidentes: permite que los médicos centren su atención en aquellas actividades para las cuales son indispensables conocimientos y capacidades que solo poseen los facultativos; mejora los tiempos de atención de los pacientes; disminuye los costos de las prestaciones de salud, etcétera(16:116). Con todo, la delegación de actividades médicas también tiene la potencialidad de crear peligros para los bienes jurídicos de los pacientes. Estos riesgos pueden tener su origen en una incorrecta selección de la enfermera o auxiliar paramédico a quien se transfiere la tarea, en un error en la entrega de la información que se requiere para ejecutar correctamente la actividad delegada, o en un control insuficiente de su ejecución(7:134). Para eliminar o disminuir hasta límites tolerables tales peligros, los médicos tienen que cumplir determinados deberes jurídicos. El inc. $2^{\circ}$ del art. 113 del Código Sanitario se refiere de manera expresa solo a uno de ellos, a saber: el de supervigilancia; pero además de este deber los facultativos han de acatar sus obligaciones de elección e instrucción. En la medida en que el médico cumpla los deberes jurídicos de elección, instrucción y supervigilancia, puede confiar en que el personal de colaboración realizará correctamente la tarea delegada. Luego, el cumplimiento de estos deberes constituye el presupuesto de la vigencia del principio de confianza en la división vertical del trabajo(17:187). 


\subsection{Los deberes de elección, instrucción y su- pervigilancia}

\subsubsection{El deber de elección}

El deber de elección comprende dos obligaciones. Por una parte, la de elegir cuidadosamente la actividad médica cuya ejecución va a ser transferida, teniendo en cuenta las condiciones físicas y psíquicas del paciente, así como los recursos humanos y materiales que existen en el recinto de salud respectivo (elección de la tarea); y por otra, la de escoger correctamente a la enfermera o al auxiliar paramédico que asumirá la tarea delegada (elección del personal)(14:167s.).

La elección de la tarea será cuidadosa cuando el médico delegue una actividad cuya realización puede llevarse a cabo por personal de colaboración, atendida su baja dificultad o peligrosidad y, además, cuando no concurren circunstancias particulares (relativas al estado de salud del paciente o a condiciones especiales de su tratamiento) que desaconsejen la delegación(14:168).

Por su parte, el deber de elección del personal implica escoger como destinatarios de la delegación, únicamente a aquellas personas de las cuales se puede esperar una ejecución cuidadosa de la tarea transferida. La importancia del cumplimiento de este deber se ve reflejada en una sentencia de la Corte Suprema de 10 de marzo de 2011, que confirmó la condena dictada en contra de una doctora a título de lesiones culposas, por haber delegado la aplicación de una inyección intravenosa en un auxiliar paramédico no capacitado para realizar el procedimiento5.

El cumplimiento del deber de elección del personal supone que la enfermera o el auxiliar paramédico tiene que contar con la calificación formal y material necesaria(18:470). La calificación formal implica que la persona cuenta con los títulos universitarios o técnicos que acreditan la posesión de determinados conocimientos ${ }^{6}$. Lo habitual será

\footnotetext{
5 Sentencia de la Corte Suprema de 10 de marzo de 2011, Rol n.o 2.285-2010 (considerandos $5^{\circ}, 6^{\circ}$ y $8^{\circ}$ de la sentencia de reemplazo).

"Ya en el "caso del tratamiento con cloroformo" del año 1952, el Tribunal Supremo Federal alemán destacó que un médico puede, en general, partir de la base de que el personal de colaboración que posee determinados títulos, cuenta con los conocimientos necesarios para realizar actividades delegadas(19).
}

que la calificación formal de enfermeras y auxiliares paramédicos sea comprobada por el departamento de recursos humanos de la respectiva clínica u hospital(14:175). El médico que pretende delegar una determinada actividad puede confiar en que dicho departamento ha realizado una correcta verificación de la calificación formal.

Ahora bien, la formación técnica o universitaria, certificada a través del respectivo título, no garantiza que la enfermera o el auxiliar paramédico esté capacitado para realizar la específica tarea delegada, puesto que esta puede no haber formado parte de los respectivos planes de estudio o módulos de prácticas. Además, los conocimientos adquiridos en la correspondiente formación se vuelven frecuentemente obsoletos, debido a los continuos avances científicos en la medicina, a nuevas posibilidades técnicas de tratamiento de ciertas enfermedades, etcétera(14:169s.). Por eso, junto con comprobar la calificación formal, es necesario que el facultativo que pretende delegar la tarea verifique la calificación material de la persona que cumple funciones de colaboración, es decir, se cerciore que esta cuenta, efectivamente, con la capacidad para enfrentar correctamente la tarea delegada. Esta aptitud concurre, en todo caso, si la persona cuenta con experiencia previa en la actividad que se le pretende transferir.

\subsubsection{El deber de instrucción}

Para que el médico pueda confiar legítimamente en que la enfermera o el auxiliar paramédico ejecutará la actividad delegada con la diligencia exigida por el Derecho, es indispensable que aquel cumpla con su deber de instrucción. Para ello, el facultativo tiene que señalar al personal de colaboración cuál es la concreta actividad que se está transfiriendo y en qué paciente esta tiene que llevarse a cabo. Con el objeto de hacer frente a los riesgos derivados de una comprensión defectuosa de las instrucciones médicas, estas han de expresarse de la manera más clara posible(20:395). Si no es factible dar por escrito la respectiva orden antes que la labor sea ejecutada, deben utilizarse métodos que disminuyan al mínimo las posibilidades de error; así, por ejemplo, pedir al personal de colaboración que repita la orden verbal recibida para así comprobar su correcta compren- 
sión. En caso de que la actividad que se pretende delegar sea poco común o tenga algún grado de peligrosidad relevante (por su propia naturaleza o por el estado o las características del paciente), el facultativo debe informar detalladamente al personal de colaboración la forma de ejecución de la tarea, de modo de garantizar su correcta realización(14:179).

\subsubsection{El deber de supervigilancia}

Los deberes jurídicos de elección e instrucción se refieren a un momento previo a la efectiva realización de la actividad delegada. Durante la ejecución de esta el facultativo tiene que cumplir con el deber de supervigilancia, previsto expresamente en el inc. $2^{\circ}$ del art. 113 del Código Sanitario.

Una primera forma de interpretar el deber de supervigilancia consistiría en entender que el médico está obligado a presenciar la ejecución de la tarea delegada; de este modo podría intervenir de inmediato en caso de advertir un error en la realización de la actividad. Sin embargo, una comprensión semejante del deber de supervigilancia haría perder todo sentido a la delegación de actividades médicas(21:1626) ${ }^{7}$. Por eso, la interpretación de la doctrina rechaza la presencia continua del médico durante la realización de la actividad transferida, con la única excepción de aquellos casos en los cuales se delegan tareas especialmente difíciles, o en los que la delegación se hace en enfermeras o en auxiliares paramédicos todavía inexpertos(14:187s.). Salvo en estos casos, los médicos pueden cumplir con su deber de supervigilancia a través de la realización de pruebas aleatorias, para así verificar la correcta ejecución de la tarea, aunque incluso se puede prescindir de semejantes pruebas si el personal de colaboración ha demostrado, en ocasiones anteriores, su capacidad para ejecutar con el cuidado debido la tarea delegada(8:6).

En el contexto de la realización de pruebas aleatorias, para así dar cumplimiento al deber de supervigilancia, se pueden presentar problemas de imputación objetiva. Porque si un médico ha

\footnotetext{
${ }^{7}$ En este orden de ideas, véase la sentencia de la Corte Suprema de 28 enero de 1999(22), la que, aunque referida a los deberes de supervigilancia que una enfermera jefa debe ejercer sobre los auxiliares paramédicos bajo su independencia, destaca que el control sobre la actividad de los subalternos no puede ser constante o ininterrumpido.
}

omitido tales pruebas y posteriormente se produce la muerte o lesión culposa de un paciente respecto del cual se ha ejecutado equivocadamente la actividad delegada, la condena penal del facultativo exigirá demostrar que, de haber realizado este las pruebas aleatorias, el resultado se hubiera evitado con una probabilidad rayana en la certeza. Si en el caso concreto se establece que la realización del control no hubiera permitido evitar el resultado con ese grado de certidumbre (porque, por ejemplo, la tarea se empezó a ejecutar incorrectamente después de efectuar el control), no será posible imputar objetivamente el resultado a la infracción del deber de supervigilancia cometida por el médico, por lo cual este no responderá penalmente a título de homicidio o lesiones imprudentes(7:301ss.). En semejantes casos solo cabría una responsabilidad penal fundada en el tipo del art. 494 n. ${ }^{\circ} 10$ del Código Penal (en adelante $\mathrm{CP}$ ), que prescinde del resultado para castigar la negligencia médica.

Ahora bien, tratándose de la delegación de actividades médicas especialmente riesgosas o de pacientes graves, el deber de supervigilancia conlleva, además, la exigencia de que el médico esté disponible para ser contactado en caso de emergencia, y pueda concurrir inmediatamente al lugar en que se está ejecutando la tarea transferida(15:61).

Finalmente, los planteamientos según los cuales el deber de supervigilancia implica que el facultativo compruebe, siempre, que la tarea delegada se ejecutó correctamente, limitan de manera inaceptable los efectos buscados con la delegación de tareas médicas(8:5).

\subsubsection{Acerca de los tipos penales aplicables en caso de infracción de los deberes de elección, instrucción y supervigilancia}

Si un médico infringe culposamente los deberes jurídicos de elección, instrucción o supervigilancia y, como consecuencia de ello, se causa o no se evita la muerte o el daño a la salud de un paciente, el facultativo podrá ser imputado por los delitos de homicidio o lesiones corporales imprudentes, en virtud de lo dispuesto en el art. 491, en relación con el art. 391 n. ${ }^{\circ} 2$ o el art. 399, todos del CP. Solo resulta aplicable el art. 399 - si el incumplimiento de los deberes mencionados oca- 
siona un daño a la salud del paciente-, puesto que los restantes tipos penales de lesiones previstos en el párrafo $3^{\circ}$ del T. VIII del Libro II del CP aluden a diversos modos de comisión típica de carácter violento ("castrare", "hiriere", "golpeare", etc.), que en ningún caso pueden equipararse a las conductas consistentes en "elegir", "instruir" o "vigilar" defectuosamente. Cualquier otra interpretación vulneraría el principio de taxatividad y certeza de las normas penales. Que en los casos de dańos a la salud, producidos como consecuencia del quebrantamiento culposo de los deberes de elección, instrucción o supervigilancia, solo se pueda recurrir a la modalidad imprudente del art. 399 del CP tiene importantes consecuencias penológicas, al resultar aplicable la norma de sanción prevista en el n. ${ }^{\circ} 2$ (y no en el n. ${ }^{\circ} 1$ ) del art. 490 del mismo cuerpo de leyes.

\subsection{Acerca de las actividades médicas indele- gables en el personal de colaboración}

Aunque el inc. $2^{\circ}$ del art. 113 del Código Sanitario no establece ninguna limitación expresa respecto de las actividades propias del ejercicio de la medicina susceptibles de ser delegadas en el personal de colaboración, no todas ellas pueden ser transferidas. Y es que la finalidad de la delegación consiste en que los facultativos concentren su atención y tiempo en aquellas actividades para las cuales son indispensables conocimientos y capacidades que poseen únicamente los médicos; respecto de tales actividades no cabe delegación alguna. El médico que las transfiera comete un error de tratamiento(23:6s.) y no actúa bajo el amparo del principio de confianza.

Responder cuáles son las actividades médicas indelegables no es un asunto sencillo. Algunos autores han indicado que los límites de la delegación no pueden establecerse exclusivamente conforme a criterios jurídicos(14:17), y que el carácter de indelegable o no de una determinada tarea debe considerar las pautas que la ciencia y praxis médicas aporten en cada caso concreto(7:167s.). Sin embargo, teniendo en cuenta la importancia de los bienes jurídicos en juego, el Derecho no puede renunciar a su misión de fijar criterios mínimos que permitan aclarar qué actividades no pueden ser delegadas(14:17).
Según la doctrina, no son delegables aquellas tareas que, por su dificultad, peligrosidad para el paciente o imprevisibilidad de las reacciones que puedan ocasionar, presuponen un conocimiento técnico médico-profesional del que solo disponen los facultativos y que, en consecuencia, tienen que ser ejecutadas personalmente por estos(23:6). Mientras más difícil o peligrosa sea la tarea, o mientras más imprevisibles sean las reacciones que esta pueda causar en la salud del paciente, más cerca estaremos de una actividad indelegable.

Respecto de si se puede delegar una determinada actividad médica y en qué condiciones cabe hacerlo, es posible trazar círculos concéntricos (7:168). El primer círculo está conformado por el ámbito de tareas reservadas exclusivamente a los médicos; el segundo, por aquellas actividades que pueden delegarse en personal de colaboración, siempre y cuando exista una supervisión médica directa; el tercero, por aquellas tareas cuya ejecución se puede transferir y en las que el facultativo solo tiene el deber de realizar pruebas aleatorias o estar disponible para ser contactado en caso de emergencia. En el primer círculo concéntrico se sitúan aquellas tareas que conforman el "núcleo esencial” de la función médica, esto es, la adopción de decisiones relativas al diagnóstico del paciente y a su tratamiento, así como la ejecución de medidas diagnósticas y de tratamiento que, por su dificultad técnica o riesgo elevado, exigen un conocimiento médico-teórico y una experiencia médico-práctica $(7: 168)^{8}$. En un caso conocido por la Corte de Apelaciones de Valparaíso se condenó civilmente al Servicio de Salud Viña del Mar-Quillota por haber extraído una enfermera sangre desde la arteria yugular de un lactante, lo que le provocó un neumotórax fatal; tal procedimiento correspondía al núcleo esencial de la función médica, siendo por tanto indelegable?

\subsection{Acerca de las actividades propias del per- sonal de colaboración}

Así como existen actividades exclusivamente médicas y por tanto indelegables, hay tareas propias

\footnotetext{
${ }^{8}$ Así, por ejemplo, no es delegable la realización de una ecografía, la interpretación de una electrocardiografía ambulatoria, la colocación de un catéter o la punción en una vía venosa central.

9 Sentencia de 27 de diciembre de 2006, Rol n. ${ }^{\circ}$ 600-2006, citada por Cárdenas y Moreno(24:112s.).
} 
o exclusivas del personal de colaboración. Estas no tienen su origen en una delegación de actividades médicas basada en el inc. $2^{\circ}$ del art. 113 del Código Sanitario, sino que constituyen labores respecto de las cuales el personal de colaboración es competente originariamente.

Las tareas para cuya realización enfermeras y auxiliares paramédicos tienen una competencia originaria están descritas (de manera más o menos detallada) en el orden extrapenal. Así, conforme al inc. $4^{\circ}$ del art. 113 referido, las enfermeras tienen a su cargo, entre otras funciones, la gestión del cuidado en lo relativo a la promoción, mantención y restauración de la salud del paciente(25). Por su parte, el art. $7^{\circ}$ del Reglamento para el ejercicio de las profesiones auxiliares de la medicina, odontología, química y farmacia y otras, detalla cuáles son las tareas propias de los auxiliares paramédicos de enfermería. De este modo, el personal de colaboración se encarga de alimentar a los pacientes, controlar sus signos vitales, suministrarles medicamentos, ejecutar técnicas de higiene y confort, etcétera. Como las tareas propias del personal de colaboración no se refieren a actividades originariamente médicas que les hayan sido delegadas en virtud de lo previsto en el inc. $2^{\circ}$ del art. 113 del Código Sanitario, el control respecto de su correcta ejecución no recae en los facultativos, sino en las enfermeras supervisoras o enfermeras jefes de equipo o unidad (así se desprende, por lo demás, de las secciones IV y V de la Norma General Técnica n. ${ }^{\circ} 1$ sobre organización funcional de enfermería en los establecimientos asistenciales de los servicios de salud ${ }^{10}$ ).

\section{Conclusiones}

En el ámbito de la división horizontal del trabajo, los médicos pueden confiar en que sus colegas realizarán sus tareas con el cuidado debido. La vigencia del principio de confianza en la división horizontal de labores presupone una clara delimitación de ámbitos de responsabilidad, en la

\footnotetext{
${ }^{10}$ Fijada mediante la resolución exenta n. ${ }^{\circ} 111$ de 07.02.1994 del Ministro de Salud. En una sentencia de 11 de marzo de 1998, la Corte de Apelaciones de Santiago, en la causa Rol n. ${ }^{\circ}$ 15.282-1997, confirmó la condena dictada en contra de una enfermera jefe de una unidad de cuidados intensivos pediátrica, que no controló que los auxiliares paramédicos de la unidad conectaran a un bebé que sufría crisis de apnea a un monitor, lo que impidió detectar oportunamente un paro cardio respiratorio (CL/JUR/1190/1998).
}

que puede ser especialmente relevante la organización interna de la respectiva clínica u hospital, fijada, por ejemplo, mediante protocolos escritos. El principio de confianza ya no rige cuando resulta cognoscible que el colega no está en condiciones de cumplir correctamente con la labor que le compete, o que está infringiendo o infringirá la lex artis. La confianza tampoco opera en favor de aquel médico que ha quebrantado sus propios deberes de cuidado.

En el ámbito de la división vertical del trabajo cobra especial relevancia la delegación de actividades médicas a enfermeras o a auxiliares paramédicos, prevista en el inc. $2^{\circ}$ del art. 113 del Código Sanitario. En la medida que un médico observe los deberes jurídicos de elección, instrucción y supervigilancia, puede confiar en que la enfermera o el auxiliar paramédico ejecutarán correctamente la tarea delegada. En el contexto de la realización de pruebas aleatorias para cumplir con el deber de supervigilancia, se pueden presentar problemas de imputación objetiva. Por otra parte, si un médico infringe culposamente los deberes de elección, instrucción o supervigilancia y, como consecuencia de ello, se ocasiona o no se evita la muerte o el daño a la salud de un paciente, aquel podrá ser imputado por los delitos de homicidio o lesiones corporales imprudentes, en virtud de lo dispuesto en el art. 491, en relación con el art. 391 n. ${ }^{\circ} 2$ o el art. 399, todos del CP. Finalmente, aunque el inc. $2^{\circ}$ del art. 113 del Código Sanitario no establece ninguna limitación expresa respecto de las actividades propias del ejercicio de la medicina susceptibles de ser delegadas, no es posible la transferencia de aquellas tareas que, por su dificultad, peligrosidad para el paciente o imprevisibilidad de las reacciones que puedan ocasionar, presuponen un conocimiento técnico médico-profesional del que solo disponen los facultativos.

\section{Agradecimientos}

Este artículo ha sido desarrollado en el marco del proyecto Fondecyt de Iniciación número 1160021, "La determinación del deber de cuidado en los delitos culposos de resultado". El autor agradece el apoyo recibido a Conicyt, así como a la Universidad de Chile. 


\section{Referencias}

1. Katzenmeier C. Arbeitsteilung, Teamarbeit und Haftungs. Medizinrecht 2004; 22: 34-40.

2. Sentencia de la Corte Suprema de 20 de junio de 1996, Rol n. ${ }^{\circ}$ 33.393-1995, Fallos del Mes 1996; (451): 1228-55.

3. Mansdörfer M. Zur Theorie des Wirtschaftsstrafrechts. Heidelberg: C.F. Müller; 2011.

4. Rosas JI. Responsabilidad por imprudencia médica - Trabajo en equipo y principio de confianza. Razonamiento penal Revista de análisis jurídico 2012; (2): 31-41.

5. Sentencia del Tribunal Supremo Federal alemán de 2 de octubre de 1979, Neue Juristische Wochenschrift 1980: 649-650.

6. Schroth U. Die strafrechtliche Verantwortlichkeit des Arztes bei Behandlungsfehlern. En: Roxin C, Schroth U, (eds.). Handbuch des Medizinstrafrechts, 4a ed. Stuttgart: Boorberg; 2010: 125-60.

7. Ulsenheimer K. Arztstrafrecht in der Praxis. 5a ed. Heidelberg: C.F. Müller; 2015.

8. Duttge G. Straf-und haftungsrechtliche Fragen der arbeitsteiligen Wundtherapie: Aufgaben, Verantwortung und Haftung von Ärzten und Pflegepersonal. GMS Krankenhaushygiene Interdisziplinär 2007; 2(2): 1-8.

9. Peter A. Arbeitsteilung im Krankenhaus aus strafrechtlicher Sicht. Baden-Baden: Nomos; 1992.

10. Brose J. Aufgabenteilung im Gesundheitswesen - Horizontale und vertikale Arbeitsteilung auf klinischer und präklinischer Ebene. En: Roxin C, Schroth U, (eds.). Medizinstrafrecht. 2a ed. Stuttgart: Boorberg; 2001: 53-91.

11. Norma General Técnica n. 51 del Ministerio de Salud. Disponible en: http://www.ispch.cl/buttons/docs/Norma_General_Tecnica_N_51_2011.pdf [citado 6 Oct 2018].

12. Duttge G. Arbeitsteilige Medizin zwischen Vertrauen und strafbarer Fahrlässigkeit. Zeitschrift für Internationale Strafrechtsdogmatik [Internet] 2011 [citado 6 Oct 2018]; 5: 349-53. Disponible en: http://www.zis-online.com/dat/ artikel/2011_5_568.pdf.

13. Stratenwerth G, Kuhlen L. Strafrecht Allgemeiner Teil. 6a ed. München: Vahlen; 2011.

14. Bohne K. Delegation ärztlicher Tätigkeiten. Frankfurt am Main: Peter Lang; 2012.

15. Sachverständigenrat zur Begutachtung der Entwicklung im Gesundheitswesen. Gutachten 2007. Disponible en: http:// dipbt.bundestag.de/dip21/btd/16/063/1606339.pdf [citado 6 Oct 2018].

16. Umbreit H. Die Verantwortlichkeit des Arztes für fahrlässiges Verhalten anderer Medizinalpersonen. Frankfurt am Main: Peter Lang; 1992.

17. Wilhelm D. Strafrechtliche Fahrlässigkeit bei Arbeitsteilung in der Medizin. Juristische Ausbildung 1985: 183-88.

18. Spickhoff A, Seibl M. Haftungsrechtliche Aspekte der Delegation ärztlicher Leistungen an nichtärztliches Medizinpersonal. Medizinrecht 2008; 26: 463-473.

19. Sentencia del Tribunal Supremo Federal alemán de 10 de julio de 1952, Entscheidungen des Bundesgerichtshofes in Strafsachen 1953; (3): 91-9.

20. Stratenwerth G. Arbeitsteilung und ärztliche Sorgfaltspflicht. En: Bockelmann P, Gallas W, (eds.). Festschrift für Eberhard Schmidt zum 70. Geburtstag. Göttingen: Vandenhoeck \& Ruprecht; 1961: 383-400.

21. Ulsenheimer K. $₫ 140$ Die fahrlässige Tötung. En: Laufs A, Kern B, (eds.). Handbuch des Arztrechts. 4a ed. München: C.H. Beck; 2010: 1614-1637.

22. Sentencia de la Corte Suprema de 28 enero de 1999. Revista de Derecho y Jurisprudencia y Gaceta de los Tribunales 1999; 96(1): 60-64.

23. Bergmann K. Delegation und Substitution ärztlicher Leistungen auf/durch nichtärztliches Personal. Medizinrecht 2009; 27: $1-10$.

24. Cárdenas H, Moreno J. Responsabilidad médica - Estándares jurisprudenciales de la falta de servicio. Santiago: Abeledo Perrot-Legal Publishing Chile; 2011.

25. Milos P, Bórquez B, Larraín A. La "gestión de cuidado" en la legislación chilena: interpretación y alcance. Ciencia y enfermería 2010; 16(1): 17-29.

Recibido: 18 de octubre de 2018

Aceptado: 12 de noviembre de 2018 